\title{
Immunotherapy in breast cancer: where we stand?
}

\author{
Rosalin Mishra* \\ James L. Winkle College of Pharmacy, University of Cincinnati, 231 Albert Sabin Way, Cincinnati, Ohio
}

Accepted on September 22, 2018

\section{Editorial}

Several cellular signaling mechanisms including altered immune, inflammatory and oxidative stress response determine the fate of a breast tumor and its metastasis. Despite the development of different effective therapies over past several decades, breast cancer accounts for $14 \%$ of cancer deaths and is one of the major causes of cancer related deaths in united states and worldwide. Conventional therapies including surgery, chemo-, radiation- or targeted- therapies including endocrine therapy have initial promising results in treating patients with breast cancer, however fail to completely eradicate breast cancer and curtail its distant metastasis in several cases. This is attributed to the heterogeneous nature of the disease, tumor cell plasticity, expression of different hormone receptors, inter- and intra-tumor heterogeneity associated with different patients. The chemo- and radiation therapies are associated with toxicities and several side effects and have modest improvement in the overall survival in many cases of breast cancer patients. Continuous exposure of cancer cells to radiations over a prolonged period transforms the nearby normal cells to neoplastic ones. Multidrug resistance (MDR) remains the main impediment in treatment of patients with the advanced disease using targeted therapies. Immunotherapy is the new promising alternative treatment approach because of it relatively low toxicity effects and ability of the immune cells to identify and destroy cancer cells. Several immunotherapy modalities including immune checkpoint inhibitors, bispecific antibodies, cancer vaccines and stimulatory molecule agonists are in various phases of clinical trials which have generated mixed results in patients with breast cancer.

Most promising immunotherapy strategies are programmed cell death 1 (PD-1) monoclonal antibodies (mAbs) or PD-1 inhibitors for the treatment of PD-1 positive $(+)$ triple negative breast cancer (TNBC) patients where PD-1 is highly expressed. These antibodies suppress the immune checkpoint interaction and help the T-cells to kill cancer cells. Nivolumab was the first PD- $1 \mathrm{mAb}$ used in the clinical trial and is recently approved by Food and Drug Administration (FDA) for advanced melanoma and NSCLC patients. Nivolumab is currently in phase II clinical trial in combination with carboplatin in TNBC patients. Several anti-PD-L1 mAbs such as avelumab and atezolizumab are in various phases of clinical trial either as a single agent or in combination with targeted therapies. Based on the preliminary pre-clinical and clinical promising activity, a phase III study is ongoing with atezolizumab in presence of paclitaxel in metastatic TNBC patients. Several other anti-PD-1/anti-PDL1 mAbs which are in phase I clinical trials in combination with other targeted therapies in TNBC patients include JS001 in combination with stereotactic body radiation therapy; pembrolizumab in combination with JAK2 inhibitor, ruxolitinib phosphate; FAZ053 in combination with another anti-PD-1 antibody PDR001. Anti-PD-1 mAb, pembrolizumab has advanced to phase II or III clinical trials in combination with chemo-or other targeted therapies in patients with TNBC. There are also several ongoing clinical trials in PD-1 negative (-) breast tumors although the clinical benefits and efficacy of PD-1/PD-L1 targeted therapy is mainly associated with expression levels of PD-1 and its ligand PD-L1. Cytotoxic Tlymphocyte antigen-4 (CTLA-4) based antibodies including tremelimumab, ipilimumab are also emerging as promising immunotherapy in combination with anti-PD-1/anti-PD-L1 antibodies (durvalumab, MEDI4736, nivolumab) which are in various phases of clinical trials in HER2 negative (-) breast cancers. Another phase II trial is ongoing with anti-PD-1 antibody, nivolumab in combination with another anti-CTLA-4 antibody, ipilimumab along with CD122-based cytokine therapy in TNBC patients. All these trails will evaluate the safety and efficacy of CTLA-4 antibody in mono- or combination therapy. CTLA-4 inhibitors are known to cause a series of immune-related adverse reactions (irAEs) including colitis, thyroiditis, hypophysitis and hepatitis. However, antiPD-1 antibodies show lower incidence of irAEs in lower grade breast cancers. Other common side effects include nausea and fatigue. Lymphocyte activation gene-3 (LAG-3) is another checkpoint receptor known to regulate T-cell activity, homeostasis and play a key role in regulatory T-cells (Tregs) immuno-suppressive activity. IMP321 is a recently developed LAG-3 antibody which is in phase I and II clinical trial with paclitaxel in metastatic breast cancer patients. This combination therapy is showing initial promising results as an immunotherapy with increased number of natural killer (NK) cells, cytotoxic T-cells and no clinically significant adverse effects. Another LAG-3 targeting antibody is TSR-033 which is in phase I clinical trial either alone or in combination with antiPD-1 antibody in patients with advanced solid tumors including breast cancer. HER2 and MUC1 are well expressed antigens in breast cancer, hence are the most potent candidates for antigenspecific vaccines for treatment of breast cancer. E75, a HER2derived vaccine in combination with granulocyte-macrophage colony stimulating factor (GM-CSF) increased the disease-free survival compared to single agent, GM-CSF along with minimal systemic and local toxicities in a phase I/II study in HER2+ breast cancer patients. AE37 is another HER2-derived vaccine which is evolving as a promising immunotherapy when administered in adjuvant settings. Research also indicates that HER2-derived vaccines combined with HER2 mAb (trastuzumab) or kinase inhibitor (lapatinib) have better curative effects for patients with breast cancer. There are reports of pilot clinical studies where dendritic cell vaccines are 
administered to breast cancer patients with chemotherapy where the cancer has spread beyond the primary metastatic site. Lapuleucel-T is a peripheral-blood mononuclear cell (PBMC)-based vaccine which is well tolerated in metastatic HER2+ breast cancer patient with mild adverse effects. Theratope is a MUC1-derived vaccine that has successfully moved to phase III trial in metastatic breast cancer patients. These patients who presented a robust antibody response to this vaccine had a better overall survival than control group. Other MUC1-derived vaccines including L-BLP25 and polyethylene glycol (PEG) gold particle nanoparticles have initial positive results in the preclinical analysis require further investigation for clinical trials. Several other breast tumors associated antigens which are tested in clinical trials include sialyl-TN-KLH, telomerase, survivin, mammaglobin, mutant p53 and others. Bispecific antibodies trigger the cytolytic killing of cancer cells by targeting both the antigens on the tumor cells as well as effector immune cells. Bispecific antibodies are in very initial stage of design and preclinical analysis. Ertumaxomab, targeting both HER2 and CD3 has undergone phase II clinical trial in metastatic breast cancer patients post- endocrine or HER2 targeted therapy. Although, studies were terminated after phase II trials, this antibody had transient, reversible and mild adverse effects with $33 \%$ objective response rate in a small cohort of 15 patients. Recently, HER2Bi armed activated T-cells (HER2 BATs) are in ongoing phase I and phase II trials in combination with pembrolizumab in metastatic breast cancer patients. A recent phase II trial is ongoing to analyze the activated cytokineinduced killer armed with CD3-MUC1 bispecific antibody in advanced stage breast cancer patient. XmAb20717 is another bispecific antibody targeting both PD-1 and CTLA-4 is in very initial stage of testing in advanced solid tumors including hormone receptor positive and TNBC breast cancer patients. C-met CAR T cells are at very preliminary stage of testing as an effective immunotherapy strategy for treatments of TNBCs. There are many stimulatory molecule agonist antibodies which could potentially serve as an effective immunotherapy for breast cancer treatment. Anti-OX40 agonist antibody (9B12) is effective in reducing metastatic lesions, inducing proliferation of cytotoxic T-cells with minimal toxicity effects in breast cancer patients. Another anti-OX40 antibody (MEDI6469) is in phase I and II clinical trial in combination with radiotherapy for treatment of higher grade breast cancer. Agonist antibody targeting 4-1BB (a TNF receptor family member) is known to induce T-cell immune response is yet to be evaluated in metastatic breast cancer patients. CD40 is another TNF receptor superfamily member which plays a key role in antigen presentation and cytokine production. CP-870,893 is a CD40 agonist $\mathrm{mAb}$ which has promising clinical results in patients with advanced solid tumors and melanoma is yet to be tested in breast cancer patients. Immunotherapy is influenced by multiple factors including immune cell compositions, breast tumor histology, the tumor microenvironment, subtype of breast cancer. Although lot of research is ongoing in the field of immunotherapy with the ultimate aim to eradicate breast cancer through immune mediated cytotoxicity or cell death, there is no FDA approved immunotherapy drug available for breast cancer. Most of the immunotherapies are in very infancy stage in the breast cancer research although PD-1 and CTLA-4 checkpoint inhibitors are showing promising results but are associated with toxicity effects. Thus more pre-clinical and clinical research is required to understand the efficacy of immunotherapy in breast cancer. More investigations are required to identify the basis of breast cancer immune regulation, its role in metastasis and drug resistance for effectively targeting the breast cancer cells via immune system. Further identifying new checkpoint inhibitors, their receptors and understanding their mode of action will enhance the diversity of breast cancer immunotherapy. A better approach of combining immunotherapy with other targeted therapies including mAbs, potential antioxidants and anti-inflammatory non-sterioidal agents with other hormone therapies would be promising for treatment of breast cancer.

\section{*Correspondance to}

Rosalin Mishra

James L. Winkle College of Pharmacy,

University of Cincinnati, 231 Albert Sabin Way, Cincinnati, Ohio 45267-0514

E-mail: mishrarn@ucmail.uc.edu 